\title{
Dysregulation of IncRNAs GM5524 and GM15645 involved in high-glucose-induced podocyte apoptosis and autophagy in diabetic nephropathy
}

\author{
YAMIN FENG $^{1}$, SHENG CHEN $^{1}$, JIARONG XU ${ }^{1}$, QUN ZHU ${ }^{1}$, XIAOLONG YE ${ }^{1}$, \\ DAFA DING $^{1}$, WEIHAO YAO ${ }^{2}$ and YIBING LU ${ }^{1}$ \\ ${ }^{1}$ Department of Endocrinology, The Second Affiliated Hospital of Nanjing Medical University, Nanjing, Jiangsu 210000; \\ ${ }^{2}$ Department of Endocrinology, Baoying People's Hospital, Yangzhou, Jiangsu 225800, P.R. China
}

Received December 31, 2017; Accepted June 25, 2018

DOI: $10.3892 / \mathrm{mmr} .2018 .9412$

\begin{abstract}
Diabetic nephropathy (DN) is an important microvascular complication of diabetes, and one of the leading causes of end-stage kidney disease. However, the mechanism of the DN pathogenic process remains unclear. Recently, long non-coding (lnc)RNA dysregulation has been regarded to cause the occurrence and development of various human diseases, although the functions of lncRNAs in human DN are poorly understood. The authors' previous study using microarray analysis identified hundreds of dysregulated lncRNAs in DN, although the functions of these lncRNAs were not demonstrated. Out of those dysregulated lncRNAs, Gm5524 was significantly upregulated in response to DN, while Gm15645 was significantly downregulated in response to DN. In the present study, this result was further validated by reverse transcription-quantitative polymerase chain reaction assays, and downregulating or overexpressing Gm5524 and Gm15645 in mouse podocytes. Notably, knockdown of Gm5524 and overexpression of Gm15645 induced mouse podocyte apoptosis and decreased cell autophagy in high-glucose culture conditions. In conclusion, the results of the present study revealed the roles of lncRNAs Gm5524 and Gm15645 in high-glucose induced podocyte apoptosis and autophagy during DN, which may further the understanding of the involvement of lncRNAs in DN, and provide a potential novel therapeutic target for this disease.
\end{abstract}

Correspondence to: Dr Yibing Lu, Department of Endocrinology, The Second Affiliated Hospital of Nanjing Medical University, 121 Jiangjiayuan Road, Nanjing, Jiangsu 210000, P.R. China E-mail: luyibing2004@126.com

Dr Weihao Yao, Department of Endocrinology, Baoying People's Hospital, 3 Anyidong Road, Yangzhou, Jiangsu 225800, P.R. China E-mail: byoxhy@163.com

Key words: diabetic nephropathy, long non-coding RNAs, Gm5524 and Gm15645, apoptosis, autophagy

\section{Introduction}

Diabetes mellitus is one of the most common chronic diseases worldwide, and there were 422 million adults diagnosed with diabetes globally in 2014, according to the World Health Organization $(1,2)$. As the number of novel diagnoses is increasing, this disease is attracting increased attention. Diabetic nephropathy (DN) is one of the principal microvascular complications of diabetes, and it is highly prevalent in $30-40 \%$ of hospitalized patients with diabetes $(3,4)$. DN is additionally one of the leading causes of end-stage kidney disease, which only has an $\sim 20 \% 5$-year survival rate (5). DN has been characterized by a series of abnormal pathological alterations, including glomerular hypertrophy, mesangial proliferation, thickening of the glomerular basement membrane, and accumulation of the extracellular matrix $(6,7)$. However, the dysregulated molecules and the mechanisms involved in this manifestation of disease remain poorly understood. Therefore, a better understanding of the pathogenesis and the identification of novel factors in DN may promote the development of novel therapeutics to address this complex disease.

Over the past decades, the rapid improvement of high-throughput sequencing techniques and bioinformatics methods has led to the advent of whole human genome sequencing. Annotation of sequencing results has revealed that $<2 \%$ of the whole human genome is protein coding genes; whereas, the majority of the rest are non-coding genes, which yield numerous non-coding transcripts, including microRNAs and long non-coding RNAs (lncRNAs) (8-10). IncRNAs, novel examples of non-coding RNAs, are $>200$ nucleotides in length and lack any protein coding ability (11). Previously, studies have revealed that lncRNAs are widely expressed in almost all human tissues, and are involved in a number of important biological process, including $\mathrm{X}$ chromatin imprinting, stem cell differentiation, immune responses, cell fate decision, proliferation, and transcriptional and post-transcriptional regulation (12-14). Furthermore, dysregulation of lncRNAs has been demonstrated to contribute to the development of diverse human diseases, including types of cancer, neurological and cardiovascular diseases, and diabetes (15-17). For example, knockdown of lncRNA uc.48+ improved diabetic 
sympathetic neuropathy in type 2 diabetic rats through regulation of purinergic receptor P2X 7 expression and extracellular signal-regulated kinase signaling (18).

In the case of DN, a number of lncRNAs have been demonstrated to be dysregulated by microarray analysis. Out of these lncRNAs, the function and underlying pathways associated with certain ones have been characterized and reported (19). For example, IncRNA taurine upregulated 1 alleviates extracellular matrix accumulation by acting as an endogenous sponge for microRNA (miR)-377 and thereby relieving the inhibition of peroxisome proliferator-activated receptor $\gamma$ in DN (20). In addition, Wang et al (21) reported that lncRNA CYP4B1-PS1-001 expression was significantly downregulated in response to early DN, and CYP4B1-PS1-001 overexpression inhibited mesangial cell proliferation and fibrosis. Furthermore, lncRNA metastasis associated lung adenocarcinoma transcript 1 (MALAT1) expression is downregulated in kidney cortices from streptozotocin-induced DN cases, and decreased MALAT1 is involved in high glucose-induced podocyte injury via interacting with $\beta$-catenin (22). In the authors' previous study, lncRNA expression patterns between a DN model and $\mathrm{db} / \mathrm{m}$ control mouse kidney tissues were analyzed using microarray analysis (23). It was demonstrated that hundreds of lncRNAs are dysregulated in DN, and these lncRNAs may contribute to the pathogenesis of DN by modulating multiple molecular pathways (23). However, the functions of these lncRNAs in DN remain unclear. In the present study, the microarray results were further validated, and the function of two of these lncRNAs (Gm5524 and Gm15645) in podocytes was analyzed by loss- and gain-of function assays.

\section{Materials and methods}

Animal model and tissue specimen preparation. A total of 12 8-week-old male mice, including six C57BL/KsJ db/db mice (experimental group; average weight $46.53 \pm 1.96 \mathrm{~g}$ ) and six C57BL/KsJ m/db mice (control group; average weight $22.32 \pm 1.10 \mathrm{~g}$ ) were purchased from the Experimental Animal Center of Nanjing Medical University (Nanjing, China). Animal experiments were conducted in accordance with Nanjing Medical University guidelines and ethical standards for animal care. The mice were fed and housed in well-ventilated plastic cages with stainless steel grid tops, at $22 \pm 2^{\circ} \mathrm{C}$ and $50-60 \%$ humidity, with a 12 -h light/dark cycle and free access to water. All kidney tissues were isolated immediately from the mice when they were sacrificed at 9 weeks old, and were stored at $-80^{\circ} \mathrm{C}$. The protocol used in the present study was approved by the Committee on the Ethics of Animal Experiments of The Second Affiliated Hospital of Nanjing Medical University (Nanjing, China).

Reverse transcription-quantitative polymerase chain reaction ( $R T-q P C R)$. The total RNA from tissues and cells was extracted using TRIzol ${ }^{\circledR}$ reagent (Invitrogen; Thermo Fisher Scientific, Inc., Waltham, MA, USA), according to the manufacturer's protocol. Subsequently, $1 \mu \mathrm{g}$ total RNA was reverse-transcribed into cDNA using HiScript Q RT SuperMix (Vazyme, Piscataway, NJ, USA), and the RT conditions were $50^{\circ} \mathrm{C}$ for $15 \mathrm{~min}$ and $85^{\circ} \mathrm{C}$ for $2 \mathrm{~min}$. qPCR analysis was performed using a SYBR Green qPCR kit (Vazyme). The thermocycling conditions were as follows: Heating to $95^{\circ} \mathrm{C}$ for $5 \mathrm{~min} ; 40$ cycles of $95^{\circ} \mathrm{C}$ for $10 \mathrm{sec}$; and $60^{\circ} \mathrm{C}$ for $30 \mathrm{sec}$. The $2^{-\Delta \Delta \mathrm{Cq}}$ method was used to compare the relative expression levels of lncRNAs Gm5524 and Gm15645, and GAPDH was used as the internal control (24). The primer sequences for Gm5524 were: Forward, 5'-GTCACAGTTTCCAGTGATAGGG-3' and reverse, 5'-AAGTGAGGCACTCCAGATTAAC-3'. The primer sequences for Gm15645 were: Forward, 5'-GAACTC CAGACCTTTGGAAGAG-3' and reverse, 5'-TCTGGCGAC TTTCATCAATACA-3'. The GAPDH primer sequences were: Forward, 5'-AGAAGGCTGGGGCTCATTTG-3' and reverse, 5'-AGGGGCCATCCACAGTCTTC-3'.

Cell culture. A separate primary mouse podocyte cell line was obtained from the Mount Sinai School of Medicine (New York, NY, USA). The podocytes were cultured on type I collagen in RPMI 1640 (Thermo Fisher Scientific, Inc.) supplemented with $10 \%$ fetal bovine serum (FBS; Thermo Fisher Scientific, Inc.), $10 \mathrm{U} / \mathrm{ml}$ mouse recombinant c-interferon (PeproTech, Inc., Rocky Hill, NJ, USA) and $1 \%$ penicillin and streptomycin, under a humidified incubator with $5 \% \mathrm{CO}_{2}$ at $33^{\circ} \mathrm{C}$.

Cell transfection. The mouse Gm5524 and Gm15645 cDNA sequences were synthesized and subsequently inserted into the pCDNA3.1 vector (Invitrogen; Thermo Fisher Scientific, Inc.), and the empty pCDNA3.1 vector was used as a control. Gm5524 and Gm15645 short hairpin (sh)RNA oligos were synthesized, annealed and inserted into the pLKO vector (Sigma-Aldrich; Merck KGaA, Darmstadt, Germany). All the vectors were prepared using Midiprep kits (Qiagen $\mathrm{GmbH}$, Hilden, Germany), and $2 \mu \mathrm{g}$ plasmid were transfected into mouse podocytes with $\sim 70 \%$ density in six-well plates using Lipofectamine ${ }^{\circledR} 3000$ (Invitrogen; Thermo Fisher Scientific, Inc.), according to the manufacturer's protocol. Gm5524, Gm15645 overexpression vectors and the shRNA lentivirus were purchased from Genscript (Nanjing, China). A total of $48 \mathrm{~h}$ following transfection, the cells were selected using puromycin for $48 \mathrm{~h}$ and harvested for further RT-qPCR or western blot analysis. The sequence of scrambled (Scr) shRNA (Sigma-Aldrich; Merck KGaA; $50 \mathrm{ng} / \mu \mathrm{l}$ ) is 5'-CCTAAGGTT AAGTCGCCCTCGCTCGAGCGAGGGCGACTTAACCTT AGG-3'.

Flow cytometry apoptosis assay. Podocytes transfected with sh-Gm5524, sh-Gm15645, sh-NC vector, Gm5524 vector and Gm15645 vector were harvested $48 \mathrm{~h}$ following transfection using trypsin. The cell suspension was incubated with fluorescein isothiocyanate-Annexin $\mathrm{V}$ and propidium iodide for $15 \mathrm{~min}$ at room temperature in the dark and analyzed using a flow cytometer (FACScan ${ }^{\circledR}$; BD Biosciences, Franklin Lakes, NJ, USA) equipped with CellQuest software version 5.1 (BD Biosciences).

Evaluation of autophagy by transmission electron microscopy. Podocytes were washed and fixed in glutaraldehyde $2.5 \%$ in $0.1 \mathrm{~mol} / 1$ phosphate buffer; $\mathrm{pH} 7.4$ ) at $4^{\circ} \mathrm{C}$ overnight, post-fixed in $1 \%$ osmium tetroxide for $3 \mathrm{~h}$ at $4^{\circ} \mathrm{C}$ and dehydrated. Subsequently, the samples were embedded using a Poly/Bed $812 \mathrm{kit}$ at $60^{\circ} \mathrm{C}$ for $24-48 \mathrm{~h}$ (Polysciences, Inc., Warrington, PA, USA). Samples were sliced into 70-nm ultra-thin sections 

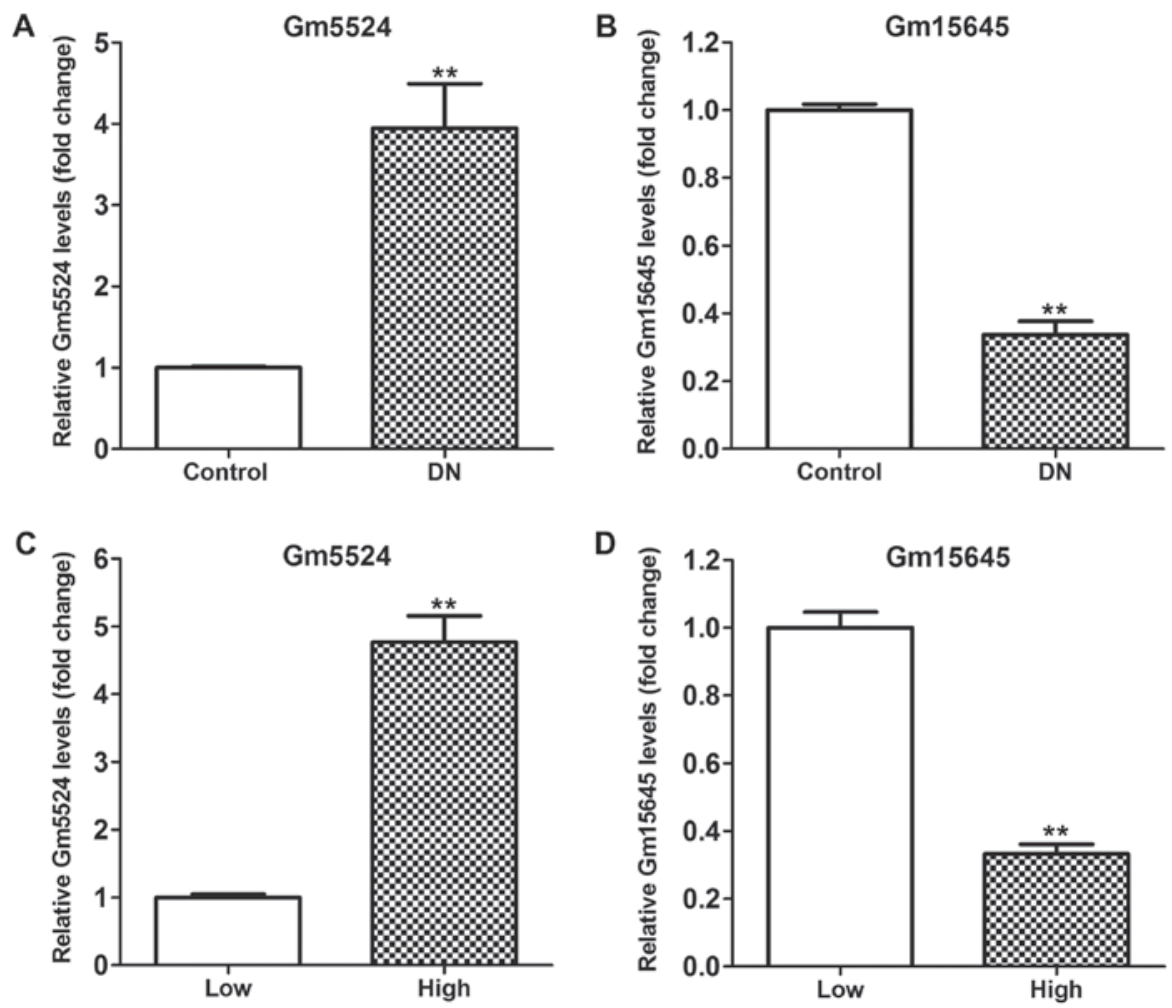

Figure 1. Gm5524 and Gm15645 expression levels in DN and podocytes. Long non-coding RNA (A) Gm5524 and (B) Gm15645 relative expression levels in DN and control mouse kidney tissues were examined using RT-qPCR. (C) Gm5524 and (D) Gm15645 relative expression levels in HG medium- or normal medium-cultured podocytes were examined using RT-qPCR. * P<0.01 vs. respective control. DN, diabetic nephropathy; HG, high glucose; RT-qPCR, reverse transcription-quantitative polymerase chain reaction.

and stained with uranyl acetate for $3 \mathrm{~min}$ at room temperature for transmission electron microscopic analysis (JEOL-1010; JEOL, Ltd., Tokyo, Japan).

Western blot analysis. The total proteins from mouse podocytes were extracted using radioimmunoprecipitation assay reagent (Beyotime Institute of Biotechnology, Haimen, China) supplemented with a protease inhibitor cocktail (Roche Molecular Diagnostics, Pleasanton, CA, USA). Subsequently, $40 \mu \mathrm{g}$ extracted protein was separated by $8-15 \%$ SDS-PAGE, and transferred to $0.22-\mu \mathrm{m}$ polyvinylidene difluoride membranes (EMD Millipore, Billerica, MA, USA). The membranes were blocked with 5\% milk in TBS with Tween-20 at room temperature for $2 \mathrm{~h}$ and subsequently incubated overnight at $4^{\circ} \mathrm{C}$ with microtubule-associated proteins 1A/1B light chain 3B (LC3)I, LC3II (cat. no. 4108; 1:1,000), autophagy protein 5 (Atg5; cat. no. $12994 ; 1: 1,000)$, ubiquitin-like modifier-activating enzyme ATG7 (Atg7; cat. no. 8558; 1:1,000), caspase 3 (cat. no. 9662; 1:1,000), cellular tumor antigen p53 (p53; cat. no. 2524; 1:1,000), apoptosis regulator BAX (Bax; cat. no. 2772; 1:1,000), apoptosis regulator $\mathrm{Bcl}-2$ (Bcl2; cat. no. 3498; 1:1,000) and GAPDH (cat. no. 5174; 1:1,000) antibodies (all from Cell Signaling Technology, Inc., Danvers, MA, USA). Membranes were subsequently incubated with horseradish peroxidase-conjugated secondary antibodies (cat. nos. 7077 and 7076; 1:5,000; Cell Signaling Technology, Inc.) for $2 \mathrm{~h}$ at room temperature. Protein bands were visualized using enhanced chemiluminescence (Bio-Rad Laboratories, Inc., Hercules, CA, USA) and a Western Blotting Detection and Imaging system (Bio-Rad Laboratories, Inc.). Enhanced chemiluminescence chromogenic substrate was quantified by densitometry (Quantity One 4.6 software; Bio-Rad Laboratories, Inc.).

Statistical analysis. All the statistical analyses were conducted using SPSS Statistics 18.0 software (SPSS, Inc., Chicago, IL, USA). One-way analysis of variance followed by the Least Significant Difference test and Student's t-test (two-tailed) were used to analyze the in vitro assay data. The data are presented as the mean \pm standard error of the mean of at least three independent assays. $\mathrm{P}<0.05$ was considered to indicate a statically significant difference.

\section{Results}

Gm5524 is upregulated and Gm15645 is downregulated in mouse DN. In the authors' previous study, IncRNA microarray analysis of an lncRNA profile in mouse DN kidney tissues and control tissues was performed (23). It was demonstrated that hundreds of IncRNAs were differentially expressed in DN tissues compared with control kidney tissues. Among these altered lncRNAs, Gm5524 was significantly upregulated and Gm15645 was significantly downregulated in DN tissues. RT-qPCR was used to further validate this in DN and control tissues, and the results demonstrated that Gm5524 was significantly upregulated and Gm15645 was significantly downregulated in DN tissues, as was the case with the microarray data $(\mathrm{P}<0.01$; Fig. $1 \mathrm{~A}$ and $\mathrm{B})$. Furthermore, podocytes were treated with high glucose to imitate the in vivo $\mathrm{DN}$ conditions, and the expression of Gm5524 and Gm15645 was examined. The results of the RT-qPCR demonstrated 

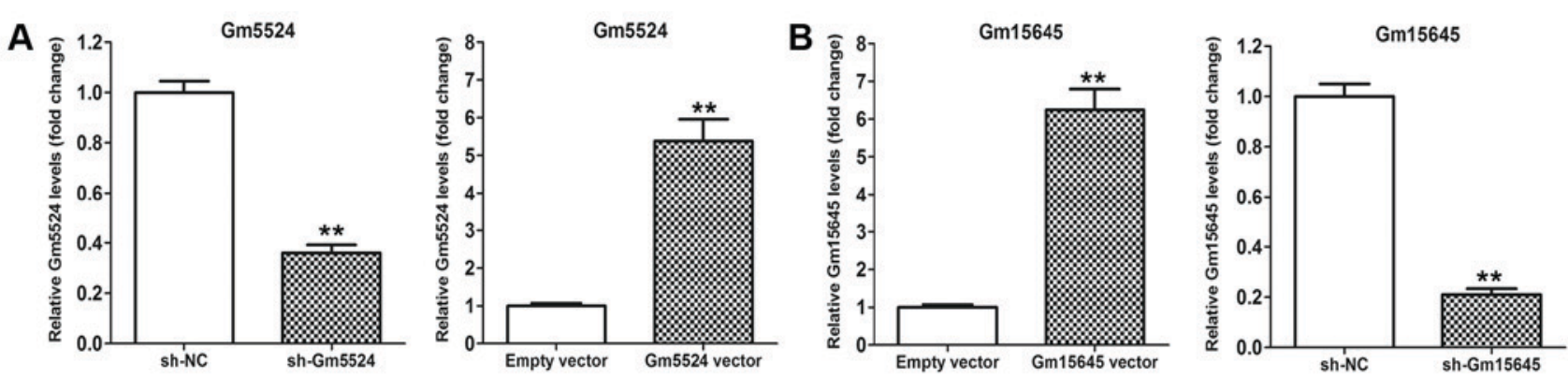

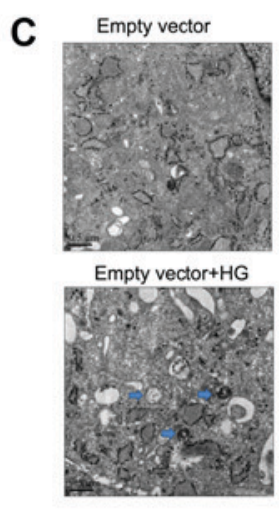

Gm5524 vector

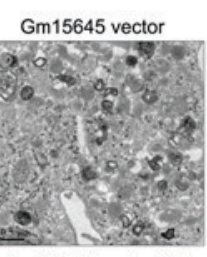

Gm5524 vector+HG

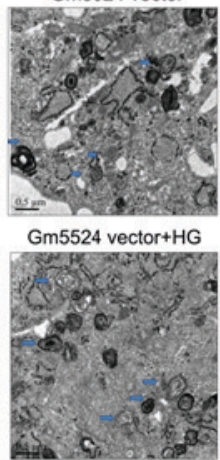

Gm15645 vector+HG

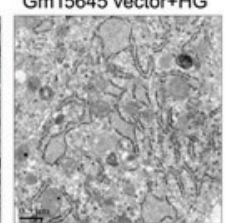

E
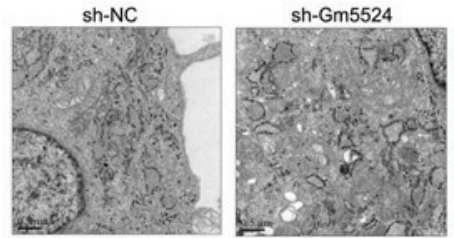

sh- $\mathrm{NC}+\mathrm{HG}$

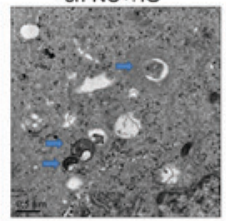

sh-Gm5524+HG

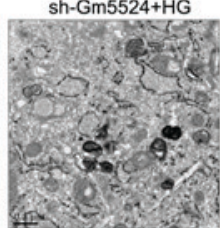

sh-Gm15645

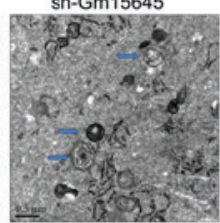

sh-Gm15645+HG

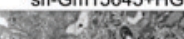

D

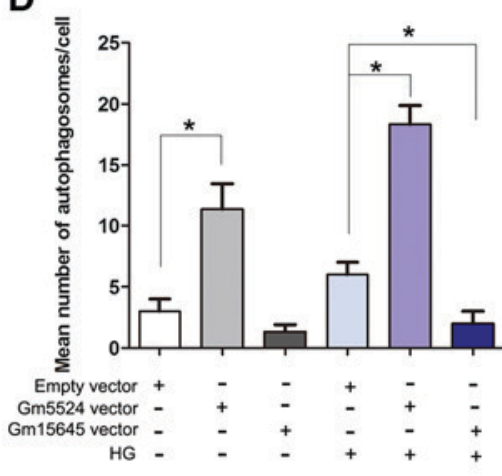

$\mathbf{F}$

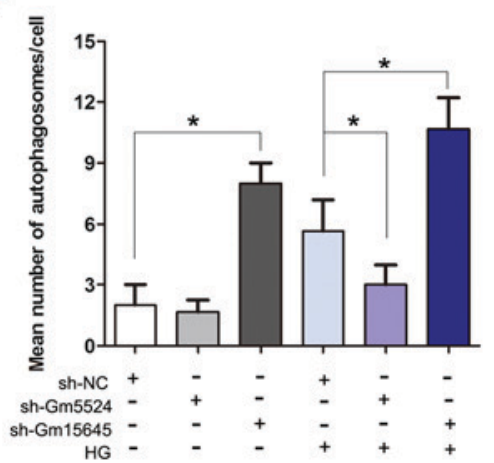

Figure 2. Effect of Gm5524 and Gm15645 on podocyte autophagy. (A) Relative expression levels of Gm5524 in empty vector-, sh-Gm5524 vector- and Gm5524 overexpression vector-treated podocytes were examined using RT-qPCR. (B) Relative expression levels of Gm15645 in empty vector-, sh-Gm15645 vector- and Gm15645 overexpression vector-treated podocytes were examined using RT-qPCR. ${ }^{* *} \mathrm{P}<0.01$ vs. respective control. (C) Cell autophagy of the empty vector-, Gm5524 and Gm15645 overexpression vector-transfected podocytes in normal or HG medium was examined using electron microscopy analysis (scale bar, $0.5 \mu \mathrm{M}$ ) and (D) quantification of these results. Blue arrows indicate autophagosomes. (E) Cell autophagy of empty vector, sh-Gm5524 vector and sh-Gm15645 vector-transfected podocytes in normal or HG medium was examined using electron microscopy analysis (scale bar, $0.5 \mu \mathrm{M}$ ) and (F) quantification of these results. Blue arrows indicate autophagosomes. ${ }^{*} \mathrm{P}<0.05$. sh, short hairpin; NC, negative control; HG, high glucose; RT-qPCR, reverse transcription-quantitative polymerase chain reaction.

that Gm5524 was additionally significantly upregulated and Gm15645 was significantly downregulated in podocytes under high glucose conditions $(\mathrm{P}<0.01$; Fig. $1 \mathrm{C}$ and $\mathrm{D})$. Additionally, specific shRNAs were designed for the two lncRNAs and transfected into mouse podocytes to knock down their expression. Furthermore, Gm5524 and Gm15645 overexpression vectors were constructed and transfected into mouse podocytes to upregulate their expression. The results of the RT-qPCR revealed that Gm5524 and Gm15645 expression was significantly deceased or increased, respectively, following transfection with shRNAs or overexpression vectors compared with control cells ( $\mathrm{P}<0.01$; Fig. 2A and B).

Gm5524 and Gm15645 affect the podocyte autophagy process. Increasing evidence has demonstrated that cell autophagy serves an important role in human diseases. To investigate whether cell autophagy may be affected by Gm5524 and
Gm15645 in DN, electron microscopy analysis was performed. Firstly, the podocytes were cultured under normal conditions or high glucose following transfection with Gm5524 and Gm15645 shRNAs or overexpression vector (Fig. 2A and B). The visualization of autophagosomes and quantification of mean autophagosome number/cell demonstrated that podocytes exhibit more electrodense inclusions and lipid granules following overexpression of Gm5524 or knockdown of Gm15645, which was more marked in high glucose-treated podocytes. In the control cells, autophagosomes were decreased or no autophagosomes were observed (Fig. 2C-F). These results suggested that the presence of autophagy may contribute to DN when Gm5524 expression is increased and Gm15645 expression is decreased.

Effect of Gm5524 and Gm15645 on mouse podocyte apoptosis. To determine whether Gm5524 and Gm15645 
A

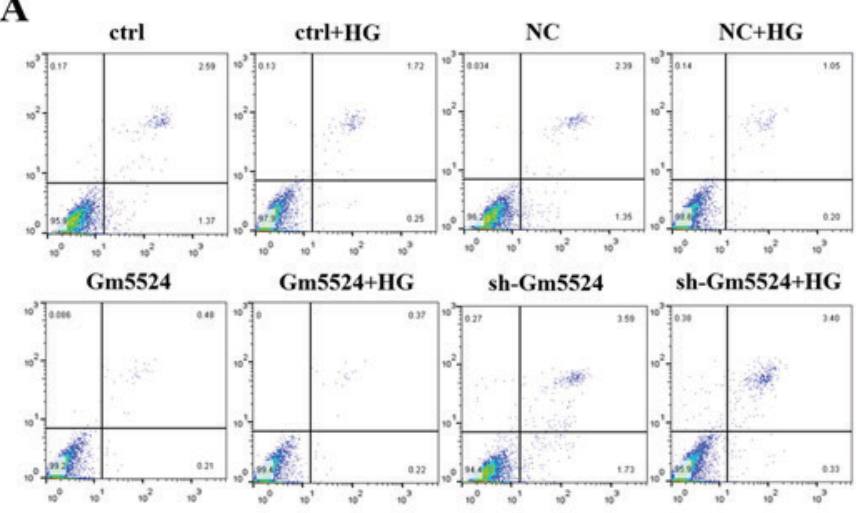

C
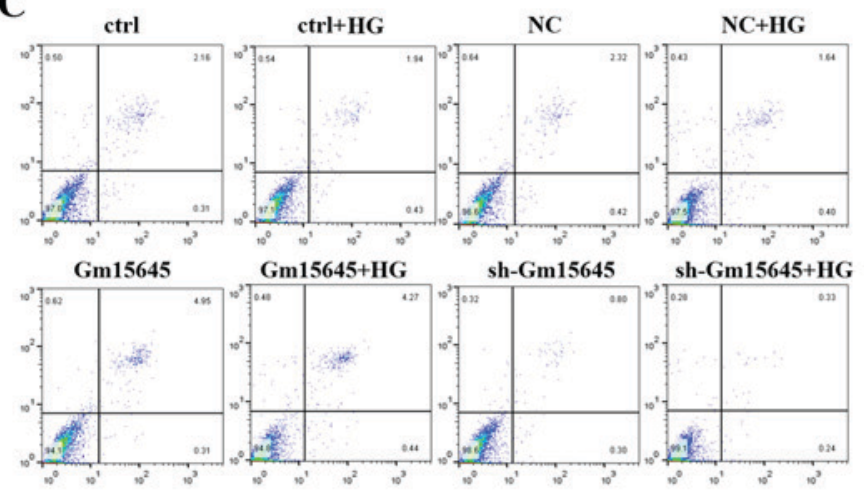

B

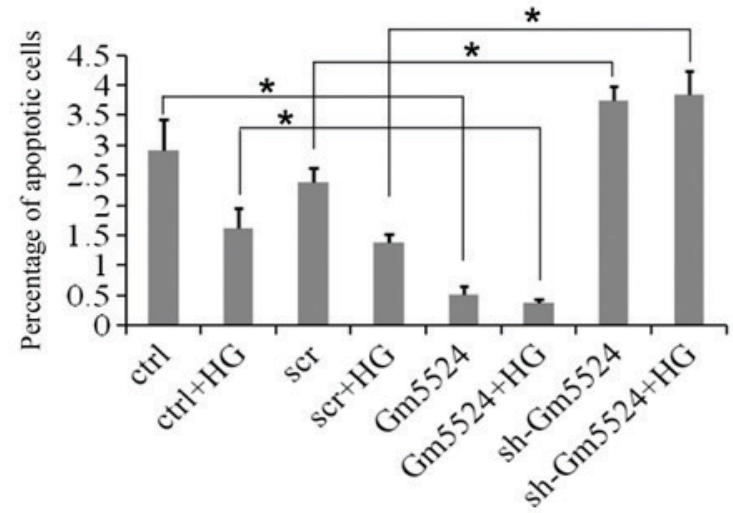

D

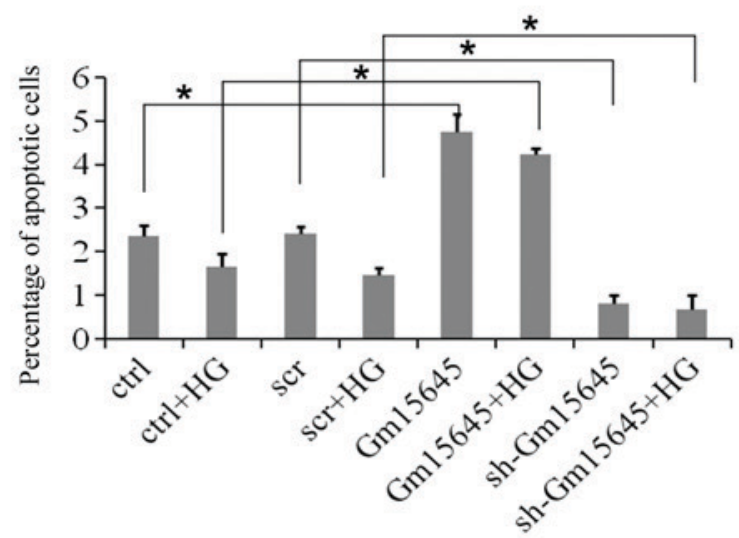

Figure 3. Effect of Gm5524 and Gm15645 on podocyte apoptosis. (A) Cellular apoptosis of empty vector-, sh-Gm5524 vector- and Gm5524 overexpression vector-transfected podocytes in normal or HG medium was examined using flow cytometry, and (B) the quantification of these results is presented. (C) Cellular apoptosis of empty vector-, sh-Gm15645 vector- and Gm15645 overexpression vector-transfected podocytes in normal or HG medium was examined using flow cytometry, and (D) the quantification of these results is presented. ${ }^{*} \mathrm{P}<0.05$. HG, high glucose; scr, scrambled; sh, short hairpin; ctrl, control; NC, negative control.

affected podocyte apoptosis, flow cytometry apoptosis assays were performed. Flow cytometry analyses of these cells demonstrated that podocytes exhibited a significantly higher apoptotic percentage following knockdown of Gm5524 expression under normal conditions, while the apoptotic percentage was significantly higher in high glucose-treated podocytes $(\mathrm{P}<0.05)$. Conversely, Gm5524 overexpression significantly decreased the podocyte apoptotic percentage under the two conditions $(\mathrm{P}<0.05$; Fig. $3 \mathrm{~A}$ and $\mathrm{B})$. Furthermore, podocytes exhibited a higher apoptotic percentage following the upregulation of Gm15645 expression under normal conditions, and the apoptotic percentage was significantly increased under high glucose conditions compared with the control; however, Gm15645 downregulation significantly decreased the podocyte apoptotic percentage under the two conditions $(\mathrm{P}<0.05$; Fig. 3C and D).

Gm5524 and Gm15645 affect the expression of apoptosis and autophagy-associated factors. To determine whether Gm5524 and Gm15645 may affect the expression levels of cellular apoptosis and autophagy-associated regulators, western blotting was performed in podocytes transfected with Gm5524 and Gm15645 shRNAs or an overexpression vector. The results demonstrated that knockdown of Gm5524 and overexpression of Gm15645 increased cleaved caspase 3, Bax and LC3I expression, while it decreased LC3II, Atg5, Atg7 and $\mathrm{Bcl} 2$ expression. Conversely, Gm5524 overexpression and
Gm15645 downregulation decreased cleaved caspase 3, Bax and LC3I expression, and increased LC3II, Atg5, Atg7 and $\mathrm{Bcl} 2$ protein expression (Fig. 4). LC3II/LC3I protein expression is a well-established biochemical assay to determine the activation of autophagy, and the present results demonstrated that the altered expression of Gm5524 and Gm15645 additionally affected the LC3II/LC3I ratio.

\section{Discussion}

The sequencing of diverse tissue samples and different cell lines has identified lncRNAs as a novel class of non-coding RNAs. Subsequently, emerging evidence has revealed that lncRNAs serve critical roles in various biological processes via regulation of target gene expression by recruiting chromatin-modifying complexes to their promoters in the nucleus, or by affecting mRNA stability and translational efficiency $(25,26)$. Recent studies have demonstrated that lncRNAs are also critical regulators of disease processes, in addition to their functional roles in normal physiological processes (27-29). In cancer, for example, lncRNAs may function as either oncogenes or tumor suppressors, and thereby regulate cancer cell growth, metastasis and drug resistance. Although lncRNAs have been associated with tumor pathogenesis, the study of lncRNAs in other human diseases requires further investigation, including in DN. Notably, certain previous studies have identified that lncRNAs are additionally involved in the development of 
A

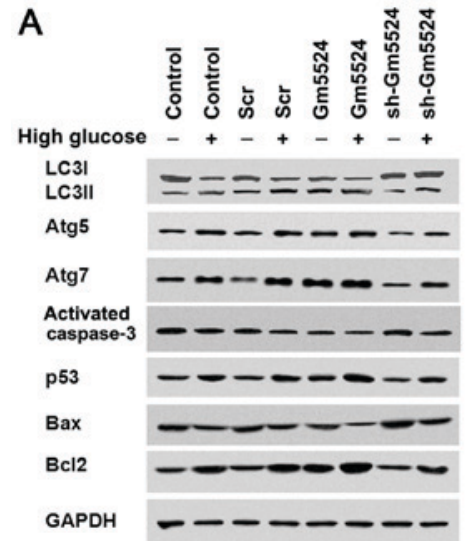

C

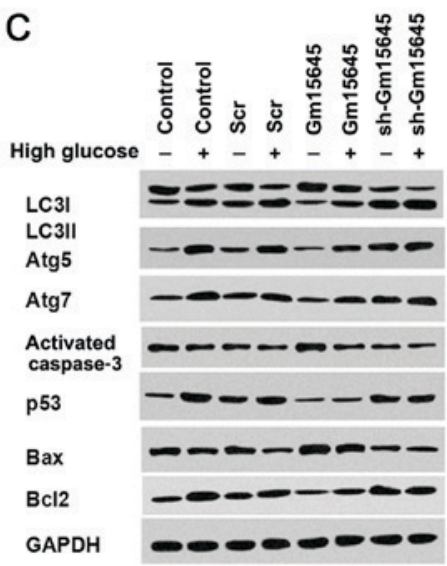

B

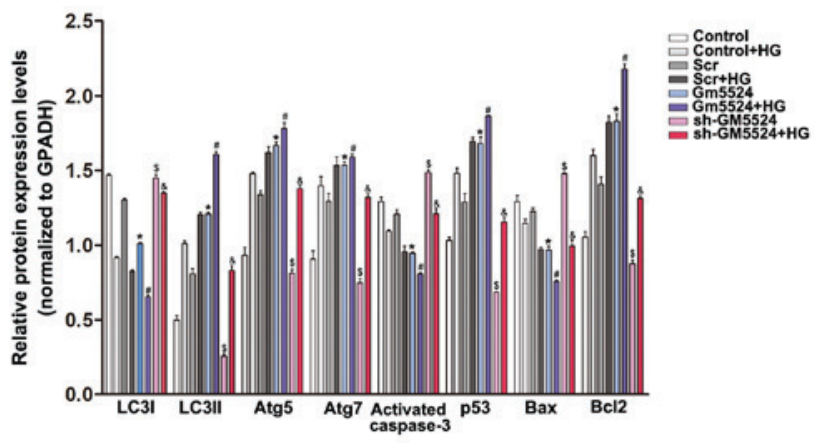

D

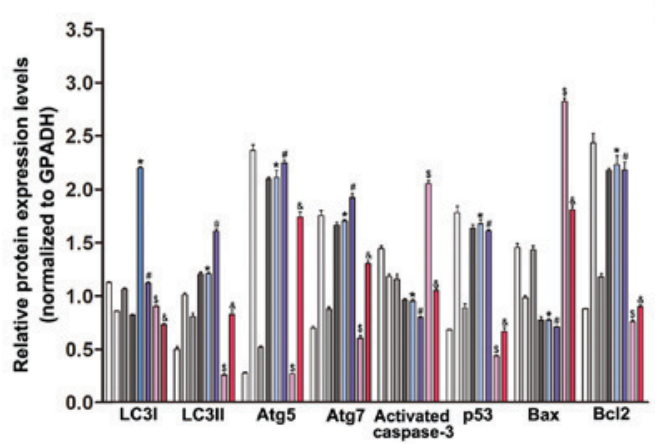

Figure 4. Effect of Gm5524 and Gm15645 on apoptosis and autophagy-associated protein expression levels in podocytes. (A) Protein expression levels of LC3I, LC3II, Atg5, Atg7, cleaved caspase 3, p53, Bax, Bcl2 and p53 were detected by western blotting in podocytes following transfection with the empty vector, sh-Gm5524 vector and Gm5524 overexpression vector under normal or HG medium conditions. (B) Densitometry data from the blots. ${ }^{*} \mathrm{P}<0.05$ vs. control; ${ }^{\prime} \mathrm{P}<0.05$ vs. control+HG; ${ }^{\mathrm{S}} \mathrm{P}<0.05$ vs. Scr; ${ }^{\circledR} \mathrm{P}<0.05$ vs. Scr+HG. (C) Protein expression levels of LC3I, LC3II Atg5, Atg7, cleaved caspase 3, p53, Bax, Bcl2 and p53 was detected by western blotting in podocytes following transfection with the empty vector, sh-Gm15645 vector and Gm15645 overexpression vector under normal or HG medium conditions. (D) Densitometry data from the blots. " $\mathrm{P}<0.05$ vs. Scr; ${ }^{\#} \mathrm{P}<0.05$ vs. Scr+HG; ${ }^{\$} \mathrm{P}<0.05$ vs. control; ${ }^{\circledR} \mathrm{P}<0.05$ vs. control+HG. LC3, microtubule-associated proteins $1 \mathrm{~A} / 1 \mathrm{~B}$ light chain $3 \mathrm{~B}$; Atg5, autophagy protein 5; Atg7, ubiquitin-like modifier-activating enzyme ATG7; p53, cellular tumor antigen p53; Bax, apoptosis regulator BAX; Bcl-2, apoptosis regulator Bcl-2; HG, high glucose; sh, short hairpin; Scr, scrambled.

diabetes and its complications (30-32). For example, lncRNA myocardial infarction associated transcript has been demonstrated to be involved in diabetes-induced microvascular dysfunction by acting as a competing endogenous RNA for miR-150-5p to antagonize its repression of vascular endothelial growth factor in retinal endothelial cells (33). In addition, plasmacytoma variant translocation 1 gene is able to increase extracellular matrix (ECM) synthesis by upregulating transforming growth factor $\beta 1$, plasminogen activator inhibitor 1 and fibronectin 1, which are the principal regulators of ECM accumulation in DN (34).

Given the importance of IncRNAs in DN, IncRNA microarray analyses were initially performed to identify the differential lncRNA expression profiles between the kidney cortices of db/db DN mice and controls, in a previous study (23). As a result, $311 \mathrm{lncRNAs}$ were demonstrated to be differentially expressed in the $\mathrm{db} / \mathrm{db}$ DN tissues, including 105 upregulated lncRNAs and 206 downregulated lncRNAs. In addition to the results of the authors' previous study (23), Wang et al (21) reported that 1,018 lncRNAs (221 upregulated and 797 downregulated) were differentially expressed in kidney tissue from $\mathrm{db} / \mathrm{db}$ mice with DN. Although the expression of hundreds of
lncRNAs may be dysregulated in DN, their underlying functional roles remain unclear. In the present study, the expression of IncRNAs was further validated in mice kidney tissues with or without DN. Out of these lncRNAs, Gm5524 was upregulated and Gm15645 was downregulated, in accordance with the IncRNA microarray data and RT-qPCR validation results. Furthermore, flow cytometry apoptosis assays suggested that downregulation of Gm5524 and overexpression of Gm15645 increased the apoptotic rate of podocytes, a feature of the early stages of DN. In addition, the electron microscopy analysis revealed that alterations in Gm5524 and Gm15645 expression were able to induce podocyte autophagy.

Cellular autophagy and apoptosis are basic cellular processes, and are essential for the maintenance of normal tissue homeostasis under physiological conditions. Disorder of these processes has been reported in diabetes and its complications (35). The results of the present study suggested that Gm5524 and Gm15645 may be involved in DN by affecting these two processes. To further investigate the molecular mechanisms underlying the involvement of Gm5524 and Gm15645 during the pathogenesis of DN, the effects of Gm5524 and Gm15645 on apoptosis 
and autophagy-associated factors were examined. It was demonstrated that the $\mathrm{Bcl} 2$ protein expression was decreased and Bax expression was increased in Gm5524-knockdown and Gm15645-overexpressing podocytes. Bcl-2 is an important anti-apoptotic protein, while Bax is an important pro-apoptotic protein. The alteration in their expression levels was consistent with the functional results. Additionally, the ratio of LC3II/LC3I protein expression is a well-established biochemical assay to determine the activation of autophagy, and the results of the present study demonstrated that the altered expression of Gm5524 and Gm15645 also affected the LC3II/LC3I ratio. These data suggested that Gm5524 and Gm15645 may affect cellular apoptotic and autophagy processes in the diabetic mouse kidney, and thus contribute to the development and progression of DN. However, further investigations are required to further elucidate the molecular mechanisms underlying the roles of Gm5524 and Gm15645 in the pathogenesis of DN.

In conclusion, the present study revealed that lncRNA Gm5524 and Gm15645 may affect podocytes apoptosis and autophagy via regulation of the $\mathrm{Bcl} 2 / \mathrm{Bax}$, and LC3/ATG signaling pathway in DN. Therefore, it was hypothesized that Gm5524 and Gm15645-mediated regulation of autophagy and apoptotic signaling pathways may be potential, useful therapeutic targets for the development of alternative treatment strategies for patients with DN.

\section{Acknowledgements}

The authors would like to thank the entire staff at the Biotech Treatment Center, The Second Affiliated Hospital of Nanjing Medical University for their excellent technical assistance with this research.

\section{Funding}

The present study was supported by grants from the National Natural Science Foundation of China (grant no. 81270896), Six Talent Peaks Project in Jiangsu Province (grant no. 2013-WSN-049; Jiangsu, China) and Foundation of Nanjing Medical University (grant no. 2015NJMUZD031).

\section{Availability of data and materials}

The datasets used or analyzed during the current study are available from the corresponding author on reasonable request.

\section{Authors' contributions}

YF and YL conceived and designed the study. YF, SC, JX, QZ and XY performed the experiments. YF, DD and WY analyzed the data. YF and DD wrote the paper. WY and YL reviewed and edited the manuscript. All authors read and approved the final version of the manuscript.

\section{Ethics approval and consent to participate}

The protocol used in the present study was approved by the Committee on the Ethics of Animal Experiments of The Second Affiliated Hospital of Nanjing Medical University.

\section{Patient consent for publication}

Not applicable.

\section{Competing interests}

The authors declare that they have no competing interests.

\section{References}

1. Katsarou A, Gudbjörnsdottir S, Rawshani A, Dabelea D, Bonifacio E, Anderson BJ, Jacobsen LM, Schatz DA and Lernmark Å: Type 1 diabetes mellitus. Nat Rev Dis Primers 3: 17016, 2017.

2. Bansal D, Gudala K, Muthyala H, Esam HP, Nayakallu R and Bhansali A: Prevalence and risk factors of development of peripheral diabetic neuropathy in type 2 diabetes mellitus in a tertiary care setting. J Diabetes Investig 5: 714-721, 2014.

3. Adeshara KA, Diwan AG and Tupe RS: Diabetes and complications: Cellular signaling pathways, current understanding and targeted therapies. Curr Drug Targets 17: 1309-1328, 2016.

4. Gregg EW, Sattar N and Ali MK: The changing face of diabetes complications. Lancet Diabetes Endocrinol 4: 537-547, 2016.

5. Magee C, Grieve DJ, Watson CJ and Brazil DP: Diabetic Nephropathy: A tangled web to unweave. Cardiovasc Drugs Ther 31: 579-592, 2017.

6. Han Q, Zhu H, Chen X and Liu Z: Non-genetic mechanisms of diabetic nephropathy. Front Med 11: 319-332, 2017.

7. Sharma D, Bhattacharya P, Kalia K and Tiwari V: Diabetic nephropathy: New insights into established therapeutic paradigms and novel molecular targets. Diabetes Res Clin Pract 128: 91-108, 2017.

8. Djebali S, Davis CA, Merkel A, Dobin A, Lassmann T, Mortazavi A, Tanzer A, Lagarde J, Lin W, Schlesinger F, et al: Landscape of transcription in human cells. Nature 489: 101-108, 2012.

9. Harrow J, Frankish A, Gonzalez JM, Tapanari E, Diekhans M, Kokocinski F, Aken BL, Barrell D, Zadissa A, Searle S, et al: GENCODE: The reference human genome annotation for The ENCODE Project. Genome Res 22: 1760-1774, 2012.

10. Derrien T, Johnson R, Bussotti G, Tanzer A, Djebali S, Tilgner H, Guernec G, Martin D, Merkel A, Knowles DG, et al: The GENCODE v7 catalog of human long noncoding RNAs: Analysis of their gene structure, evolution, and expression. Genome Res 22: 1775-1789, 2012.

11. Ponting CP, Oliver PL and Reik W: Evolution and functions of long noncoding RNAs. Cell 136: 629-641, 2009.

12. Mercer TR, Dinger ME and Mattick JS: Long non-coding RNAs: Insights into functions. Nat Rev Genet 10: 155-159, 2009.

13. Guttman M, Donaghey J, Carey BW, Garber M, Grenier JK, Munson G, Young G, Lucas AB, Ach R, Bruhn L, et al: LincRNAs act in the circuitry controlling pluripotency and differentiation. Nature 477: 295-300, 2011.

14. Fatica A and Bozzoni I: Long non-coding RNAs: New players in cell differentiation and development. Nat Rev Genet 15: 7-21, 2014.

15. He X, Ou C, Xiao Y, Han Q, Li H and Zhou S: LncRNAs: Key players and novel insights into diabetes mellitus. Oncotarget 8: 71325-71341, 2017

16. Shi X, Sun M, Liu H, Yao Y and Song Y: Long non-coding RNAs: A new frontier in the study of human diseases. Cancer Lett 339: 159-166, 2013.

17. Bhan A and Mandal SS: Long noncoding RNAs: Emerging stars in gene regulation, epigenetics and human disease. ChemMedChem 9: 1932-1956, 2014.

18. Wu B, Zhang C, Zou L, Ma Y, Huang K, Lv Q, Zhang X, Wang S, Xue Y, Yi Z, et al: LncRNA uc.48+ siRNA improved diabetic sympathetic neuropathy in type 2 diabetic rats mediated by P2X7 receptor in SCG. Auton Neurosci 197: 14-18, 2016.

19. Allison SJ: Diabetic nephropathy: A lncRNA and miRNA megacluster in diabetic nephropathy. Nat Rev Nephrol 12: 713, 2016.

20. Duan LJ, Ding M, Hou LJ, Cui YT, Li CJ and Yu DM: Long noncoding RNA TUG1 alleviates extracellular matrix accumulation via mediating microRNA-377 targeting of PPAR $\gamma$ in diabetic nephropathy. Biochem Biophys Res Commun 484: 598-604, 2017. 
21. Wang M, Wang S, Yao D, Yan Q and Lu W: A novel long non-coding RNA CYP4B1-PS1-001 regulates proliferation and fibrosis in diabetic nephropathy. Mol Cell Endocrinol 426: 136-145, 2016.

22. Hu M, Wang R, Li X, Fan M, Lin J, Zhen J, Chen L and Lv Z: LncRNA MALAT1 is dysregulated in diabetic nephropathy and involved in high glucose-induced podocyte injury via its interplay with $\beta$-catenin. J Cell Mol Med 21: 2732-2747, 2017.

23. Chen S, Dong C, Qian X, Huang S, Feng Y, Ye X, Miao H, You Q, Lu Y and Ding D: Microarray analysis of long noncoding RNA expression patterns in diabetic nephropathy. J Diabetes Complications 31: 569-576, 2017.

24. Livak KJ and Schmittgen TD: Analysis of relative gene expression data using real-time quantitative PCR and the 2(-Delta Delta C(T)) method. Methods 25: 402-408, 2001.

25. Tsai MC, Manor O, Wan Y, Mosammaparast N, Wang JK, Lan F, Shi Y, Segal E and Chang HY: Long noncoding RNA as modular scaffold of histone modification complexes. Science 329: 689-693, 2010

26. Wang KC and Chang HY: Molecular mechanisms of long noncoding RNAs. Mol Cell 43: 904-914, 2011.

27. Zhang J, Zhu Y and Wang R: Long noncoding RNAs in respiratory diseases. Histol Histopathol: 11966, 2018.

28. Sallam T, Sandhu J and Tontonoz P: Long noncoding RNA discovery in cardiovascular disease: Decoding form to function. Circ Res 122: 155-166, 2018

29. Saha P, Vermas S, Pathak RU and Mishra RK: Long noncoding RNAs in mammalian development and diseases. Adv Exp Med Biol 1008: 155-198, 2017.
30. Leung A and Natarajan R: Long noncoding RNAs in diabetes and diabetic complications. Antioxid Redox Signal: Oct 30, 2017 (Epub ahead of print)

31. Sun Y and Liu YX: LncRNA HOTTIP improves diabetic retinopathy by regulating the p38-MAPK pathway. Eur Rev Med Phamacol Sci 22: 2941-2948, 2018.

32. Zhang N, Geng T, Wang Z, Zhang R, Cao T, Camporez JP, Cai SY, Liu Y, Dandolo L, Shulman GI, et al: Elevated hepatic expression of H19 long noncoding RNA contributes to diabetic hyperglycemia. JCI Insight 3: pii: 120304, 2018.

33. Yan B, Yao J, Liu JY, Li XM, Wang XQ, Li YJ, Tao ZF, Song YC, Chen Q and Jiang Q: IncRNA-MIAT regulates microvascular dysfunction by functioning as a competing endogenous RNA. Circ Res 116: 1143-1156, 2015.

34. Alvarez ML and DiStefano JK: Functional characterization of the plasmacytoma variant translocation 1 gene (PVT1) in diabetic nephropathy. PLoS One 6: e18671, 2011.

35. Hamzawy M, Gouda SAA, Rashid L, Attia Morcos M, Shoukry H and Sharawy N: The cellular selection between apoptosis and autophagy: Roles of vitamin D, glucose and immune response in diabetic nephropathy. Endocrine 58: 66-80, 2017.

This work is licensed under a Creative Commons Attribution-NonCommercial-NoDerivatives 4.0 International (CC BY-NC-ND 4.0) License. 